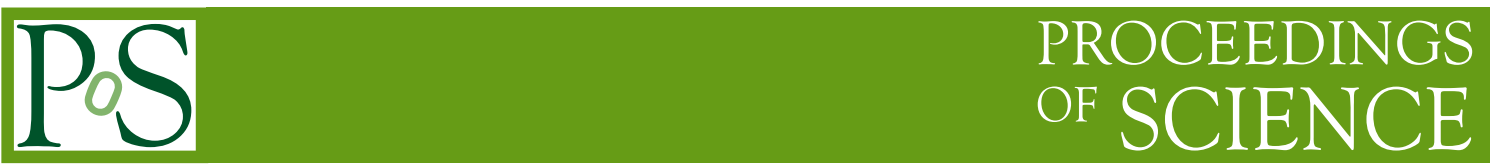

\title{
Calibrating the DUNE LArTPC detectors for precision physics
}

\author{
Viktor Pěč for the DUNE collaboration \\ Department of Physics and Astronomy, The University of Sheffield, \\ Hicks Building, Hounsfield Road, Sheffield, United Kingdom \\ E-mail: v.pec@sheffield.ac.uk
}

The Deep Underground Neutrino Experiment (DUNE) is an international collaboration focused on studying neutrino oscillation over a long baseline $(1300 \mathrm{~km})$. DUNE will make use of a near detector and $O(\mathrm{GeV})$ neutrino beam originating at Fermilab in Batavia, IL, and a far detector operating $1.5 \mathrm{~km}$ underground at the Sanford Underground Research Facility in Lead, South Dakota. The near and far detectors will use the LArTPC (Liquid Argon Time Projection Chamber) technology to image neutrino interactions. In order to make precise physics measurements at DUNE, such as the amount of CP violation in the neutrino sector, it is essential to be able to accurately reconstruct particle energies and other kinematic quantities; this in turn necessitates an extensive calibration program for DUNE's LArTPC detectors. In this presentation, we describe the requirements for calibrating the DUNE detectors, emphasizing the challenges of massive multikiloton LArTPC detectors which are to operate for multiple years deep underground. A preliminary DUNE detector calibration program, including use of both dedicated calibration hardware and cosmogenic/beam-induced calibration sources, is presented. First results on detector calibration at the ProtoDUNE-SP prototype detector located at CERN, and associated impact on calibrations at the DUNE far detector, are also emphasized.

40th International Conference on High Energy physics - ICHEP2020

July 28 - August 6, 2020

Prague, Czech Republic (virtual meeting) 


\section{Introduction}

The Deep Underground Neutrino Experiment (DUNE) has ambitious physics goals which include determination of the CP-violating phase in neutrino oscillations, determination of neutrino mass ordering, detection of neutrinos from supernova bursts, searches for nucleon decay, and more. DUNE has chosen Liquid Argon Time Projection Chamber (LArTPC) as its main detector technology.

To achieve such goals, DUNE will be facing unprecedented challenges by operating kilotonscale detectors with liquid argon (LAr), over an extended period of time of at least 10 years. It will be crucial to properly understand the detector response and its variation across the large volume and over time. This will require an extensive calibration programme. The development of the strategy for calibrations of the DUNE LArTPC detectors is described in this contribution.

DUNE will be operating detectors at two distances from the neutrino beam source at Fermilab. The Near Detector (ND) will be located just downstream of the source and will utilise 3 different technologies, a LArTPC, a gaseous argon TPC, and a tracker. The Far Detector (FD) will be located approximately $1300 \mathrm{~km}$ further downstream, at SURF in South Dakota, $1480 \mathrm{~m}$ underground. It will consist of 4 modules out of which at least 3 of those will be based on LArTPC technology and will have total mass of $17 \mathrm{kt}$ ( $10 \mathrm{kt}$ in the fiducial volume) of LAr in each.

In a LArTPC, charged particles create free electrons by ionisation of argon. These electrons are then drifted by an electric field towards readout anode planes. By combining the 2D position determination at the anode plane with drift time measurements, a 3D image of the event can be reconstructed. In the first DUNE single-phase LArTPC module, ionisation charge will be drifted horizontally by an electric field of $500 \mathrm{~V} / \mathrm{cm}$, with maximum drift distance of $3.5 \mathrm{~m}$. The 2D localisation at the anode plane is achieved by means of 3 layers of wires of different orientation. Further details about the DUNE design can be found in [1].

\section{Calibration Strategy}

Convincing physics measurements will require a demonstration that the overall detector response is well understood. One of the main focuses will be on uncertainties in energy measurements. Neutrino oscillation physics is driving the requirements on uncertainty in the energy scale for leptons and hadrons to the $2 \%$ and $5 \%$ level near $1 \mathrm{GeV}$. Requirements on the low energy scale at $O(\mathrm{MeV})$ are driven by the supernova neutrino burst programme, where the resolution of energy reconstruction is required to be $20 \%-30 \%$. Requirements from other physics goals, e.g. searches for baryon number violating processes and measurements of atmospheric neutrinos, are also being evaluated.

One aim of the current DUNE calibration programme is to determine to what level we need to understand the correlation between the low-level detector properties and the detector response to various events in order to reach the required sensitivities of the physics measurements. We are developing techniques to determine low-level detector parameters like electron drift velocity, electron lifetime, electron-ion recombination, etc., and to also measure the detector response to interactions from sources with well known energy spectra. The use of several standard candles is being investigated. This list includes but is not limited to stopping power of through-going muons 
and stopping muons, Michel electrons from muon decays, delta-ray electrons, $\pi^{0}$ decays, neutron capture, and decays of radioactive isotopes.

We will have multiple sources readily available for the purpose of calibration such as cosmic muons, neutrino beam events, interactions of atmospheric neutrinos, and intrinsic radioactive isotopes. In addition, dedicated calibration devices are being designed such as an ionisation laser system and a pulsed neutron source. Use of deployable radioactive source $\mathrm{Cf} / \mathrm{Ni}$ emitting $9 \mathrm{MeV}$ gamma photons is also being considered. Selected sources are discussed in the following subsections.

Important near-term goals are the inclusion of realistic detector response calibrations in simulation-based analyses and the optimisation of the interplay between strategies for intrinsic and hardware calibrations. Both tasks will help assess analysis needs and calibration strategies as the collaboration moves forward in its construction and installation phases.

\subsection{Cosmic Ray Muons}

Cosmic ray muons are valuable sources for characterisation of detector response. Electrons from muon decays and gammas from neutral pions have characteristic energy spectra and are commonly used as standard candles. Detector performance at the low energy scale can also be validated using delta-ray electrons. Ionisation losses by muon tracks can be used to measure electron lifetime and electron-ion recombination. The straight tracks can also be used for alignment of individual TPC components and to help constrain electric field distortions.

Dedicated Monte Carlo simulations were performed for the single-phase FD module. There will be about 4,700 cosmic ray muons entering the TPC every day with mean energy of $282 \mathrm{GeV}$. Only about 90 muons per day will stop in the detector. Figure 1a shows the muon energy spectrum.

The main challenge will be the relatively low rate of muon events in the far detector due to the large depth of the experiment, in contrast with a similar detector on or near the surface. Low statistics will complicate the ability to determine variations of the calibration parameters on short time scales and throughout the TPC volume. Studies are being conducted in order to understand at what level of spatial and temporal granularity measurements can be done with a sufficient precision.

An example of such studies is the measurement of electron lifetime. Figure $1 \mathrm{~b}$ demonstrates the effect of the finite electron lifetime on the measured energy loss rates as a function of the position along the drift direction. There is a clear decrease of the measured rate for depositions further away from anode planes.

\section{$2.2{ }^{39} \mathrm{Ar}$}

The most abundant intrinsic radioactive isotope will be ${ }^{39} \mathrm{Ar}$. This isotope is naturally present in liquid argon with expected activity of $\sim 1 \mathrm{~Bq} / \mathrm{kg}$. Its beta decay with Q-value of $565 \mathrm{keV}$ will provide a well-defined energy spectrum. MicroBooNE found evidence of the decays in LArTPC carried out studies of the reconstructed energy spectrum and signal shape [2].

There are several advantages to this intrinsic source. It provides high statistics and it is uniformly distributed throughout the TPC volume, allowing for high-granularity time-dependent detector calibrations. The measured energy spectrum is sensitive to electron lifetime, electron-ion recombination, and to the electronics noise. 


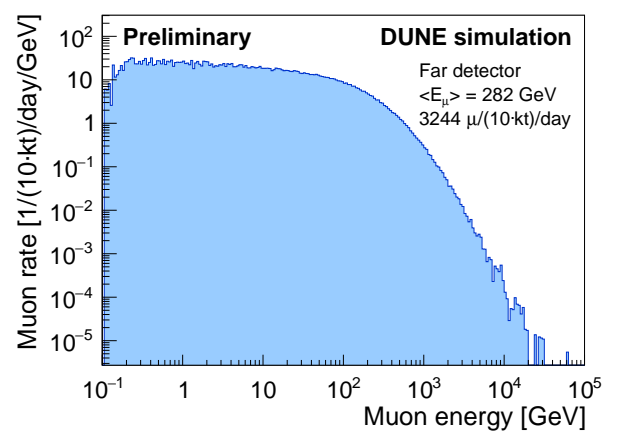

(a)

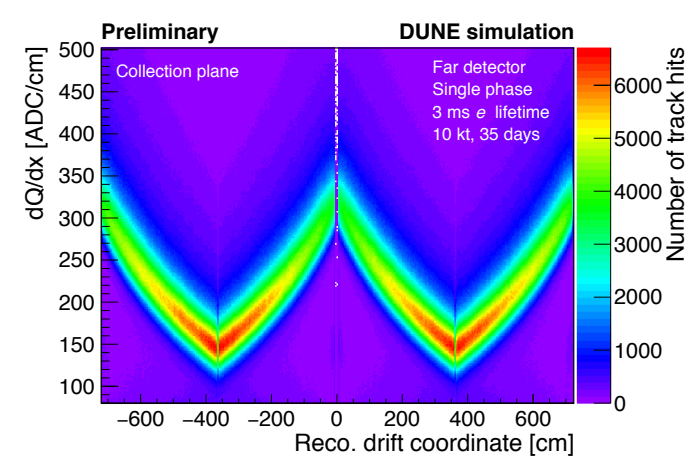

(b)

Figure 1: Results of MC simulations of cosmic ray muons at the DUNE FD. (a) Spectrum of energies of the muons reaching the TPC. About 3,200 muons a day reach the 10-kt fiducial volume, which translates to about 4,700 muons in the full active volume. (b) Example of the effect of electron lifetime on the measured charge. The electron lifetime was set to $3 \mathrm{~ms}$ in the simulations. $\mathrm{dQ} / \mathrm{dx}$ is the reconstructed ionisation charge per unit track length. The plot clearly shows variations along the drift direction. Positions of readout anode planes are at around $-730 \mathrm{~cm}, 0 \mathrm{~cm}$, and $730 \mathrm{~cm}$. Positions of cathode planes are at around $-365 \mathrm{~cm}$ and $365 \mathrm{~cm}$.

There are also some challenges associated with this source. Due to its nature, the time of the decay will be unknown and therefore it will not be possible to reconstruct its location along the drift direction, and any variations would be averaged. The relatively low Q-value limits the scope of the possible measurements. The high rate of events in the detector $\left(O\left(10^{7}\right) \mathrm{Bq} / 10 \mathrm{kt}\right)$ will require dedicated solutions for the triggering and data acquisition systems.

Use of another isotope, ${ }^{42} \mathrm{Ar}$, is also being considered. Although much less abundant than ${ }^{39} \mathrm{Ar}\left(\sim 10^{2} \mathrm{~Bq} / 10 \mathrm{kt}\right)$, its decay daughter ${ }^{42} \mathrm{~K}$ has a higher Q-value of $3.5 \mathrm{MeV}$, which would nicely expand on the reach of ${ }^{39} \mathrm{Ar}$.

\subsection{Neutral Pion Decays}

Decays of $\pi^{0}$ are a valuable standard candle to constrain the energy scale for electromagnetic showers. Measurement of the reconstructed invariant mass from the two decay photons in LArTPC was demonstrated by MicroBooNE [3].

Characteristics of $\pi^{0}$ events from both the neutrino beam interactions and cosmic muon interactions in the DUNE FD are being studied using Monte Carlo simulations. The major challenges with this source will be the overall low statistics and difficult event selection in events with large, busy hadronic showers.

\subsection{Ionisation Laser System}

The primary purpose of the ionisation laser system is to provide an independent, fine-grained estimate of the E field in space or time, which is a critical parameter for physics signals as it ultimately impacts the spatial resolution and energy response of the detector. The track like traces also allow the proper alignment of the TPC components. Its possible use for electron lifetime measurement is being currently investigated. 
The laser system used by MicroBooNE [4] was used as a reference design. That system allows to point the laser beam in various directions from two fixed points outside the field cage. As an extension, DUNE is designing multiple retractable periscopes entering apertures in the top of the field cage in order to provide coverage over the much larger volume. The possibility to also direct laser beams from the region near the vertical end walls is also being considered. A mechanism would allow offsetting the laser origin points horizontally so that the shadowing effect from the vertical field cage profiles is further reduced. The final design of the subsystems, their number and locations within the detector module are yet to be determined. A prototype design is planned to be tested in run 2 of ProtoDUNE at CERN in 2022.

\subsection{Pulsed Neutron Source}

Another dedicated hardware tool being designed for calibrations is the Pulsed Neutron Source. Neutrons will undergo capture on argon, $n+{ }^{40} \mathrm{Ar} \rightarrow{ }^{41} \mathrm{Ar}^{*}$, and will produce a deexcitation gamma cascade with the total energy release of $6.1 \mathrm{MeV}$. The source will provide high statistics for measurements of the detector response to the neutron capture events. It will also be used to constrain the energy scale and resolution for $O(1) \mathrm{MeV}$ electrons and photons. Its possible use for electron lifetime measurement is being investigated with challenges coming from the localisation of the neutron capture along the drift direction.

Even if deployed in select locations, large coverage of the detector volume is possible due to the existence of an anti-resonance in neutron elastic scattering on ${ }^{40} \mathrm{Ar}$ which is predicted at around $57 \mathrm{keV}$. This anti-resonance makes liquid argon nearly transparent to neutrons at this energy. However, the expected average scattering length of about $30 \mathrm{~m}$ still needs experimental confirmation.

\subsection{ProtoDUNE and SBN Programme}

The DUNE calibration strategy and procedures will be heavily informed by operations of the prototype detectors at CERN, ProtoDUNE, and also by the extensive short-baseline neutrino programme at Fermilab. Two technologies of LArTPC were prototyped at CERN, single and dual phase. The single-phase detector, ProtoDUNE-SP, was exposed to particle beams in 2018, and has been collecting cosmic-ray data since then. Procedures for calibration have been developed and the detector has shown excellent performance [5].

The two prototypes provide an invaluable test bed for the development of techniques to be used in the DUNE Near and Far Detectors. Furthermore, properties of liquid argon can be measured at the same electric field and, in case of ProtoDUNE-SP, the same drift distance, and directly inform the simulation and calibration for the DUNE FD.

One of the main detector effects in the ProtoDUNE detectors is the non-uniformity of the electric field due to the accumulated space charge from to the high cosmic ray flux on the surface. Although this effect is expected to be negligible for both DUNE sites, it may complicate the transfer of the calibration techniques directly from ProtoDUNE.

In addition, three LArTPC-based experiments are being built or are already operational at Fermilab: SBND, MicroBooNE, and ICARUS. DUNE will benefit heavily from the development of techniques for calibration and characterisation of detector response, prior to its start. 


\section{Conclusions}

DUNE is developing calibration techniques in order to evaluate the detector response of its LArTPC detectors. The requirements on the performance are driven by the neutrino oscillation physics at high energies and by astrophysical neutrino physics at lower energies. Connections between the physics reaches and the requirements on calibrations of fundamental detector response are being established. Several tools which have been identified to be used to measure the main detector properties, are listed in Table 1. The ProtoDUNE detectors at CERN and the extensive LArTPC programme at Fermilab will play an important role in refining the calibration strategy and developing the calibration techniques.

\begin{tabular}{ll}
\hline Measurement & Tools \\
\hline Alignment & Cosmics, Laser \\
E-field & Cosmics, Laser \\
Electron lifetime & Cosmics, ${ }^{39} \mathrm{Ar}$, Laser, Pulsed Neutron Source \\
Recombination & Cosmics, ${ }^{39} \mathrm{Ar}$, Beam \\
Energy scale & Cosmics, Beam, Pulsed Neutron Source, ${ }^{39} \mathrm{Ar}$ \\
\hline
\end{tabular}

Table 1: Summary of main detector properties to be measured (1st column) and the respective tools to be used (2nd column). The primary tools are typeset in bold. The other tools have certain potential to do the measurement or they are capable of setting constrains complementary to other measurements.

\section{References}

[1] DUNE Collaboration, Abi, B. et al., Deep Underground Neutrino Experiment (DUNE), Far Detector Technical Design Report, Volume I: Introduction to DUNE, JINST 15 (2020) T08008 [hep-ex/2002 . 02967], Volume II: DUNE Physics, [hep-ex/2002 . 03005], Volume IV: The DUNE far detector single-phase technology, JINST 15 (2020) T08010 [hep-ex/2002 . 03010]

[2] MicroBooNE Collaboration, Acciarri, R. et al., Study of Reconstructed ${ }^{39}$ Ar Beta Decays at the MicroBooNE Detector, MICROBOONE-NOTE-1050-PUB.

[3] MicroBooNE Collaboration, Adams, C. et al., Reconstruction and measurement of Script $O(100) \mathrm{MeV}$ energy electromagnetic activity from $\pi^{0} \rightarrow \gamma \gamma$ decays in the MicroBooNE LArTPC, JINST 15 (2020) P02007 [hep-ex/1910.02166]

[4] MicroBooNE Collaboration, Adams, C. et al., A Method to Determine the Electric Field of Liquid Argon Time Projection Chambers Using a UV Laser System and its Application in MicroBooNE, JINST 15 (2020) P07010 [hep-ex/1910 . 01430]

[5] DUNE Collaboration, Abi, B. et al., First results on ProtoDUNE-SP liquid argon time projection chamber performance from a beam test at the CERN Neutrino Platform, JINST 15 (2020) P12004 [hep-ex/2007.06722] 